\title{
Factor Structure of the Bulgarian CAST: (Childhood Autism Spectrum Test)
}

\author{
Mila Vulchanova ${ }^{1,2, *}$, Liubomir Djalev ${ }^{3}$, Margarita Stankova ${ }^{3}$, Valentin Vulchanov ${ }^{1,2}$, \\ Carrie Allison ${ }^{4}$ and Simon Baron-Cohen ${ }^{4}$
}

\author{
${ }^{1}$ Language Acquisition and Language Processing Lab, Norwegian University of Science \& Technology, \\ Trondheim, Norway \\ ${ }^{2}$ Department of Language \& Literature, Norwegian University of Science \& Technology, Trondheim, Norway \\ ${ }^{3}$ New Bulgarian University, Sofia, Bulgaria \\ ${ }^{4}$ Autism Research Centre, Cambridge University, UK
}

\begin{abstract}
The number of studies addressing the latent structure of specific screening and diagnostic tools for autism spectrum conditions is still limited. The current study explored the latent structure of the Bulgarian CAST (Childhood Autism Spectrum Test) in screening for autism in the Bulgarian population. The data were analysed using factor analyses. A hierarchical model with two primary factors yielded best data fit. This structure is consistent with the two main categories adopted in the DSM- 5 and current accounts of the range of conditions broadly falling into two main clusters of symptoms. These results confirm the validity of CAST as a tool for screening for autism spectrum conditions in other cultures and support the construct validity of the model and criteria included in the DSM-5. Furthermore, our results demonstrate how factor analysis can be exploited to reveal the underlying structure of screening tools aimed at capturing the behavioural traits of deficit phenotypes.
\end{abstract}

Keywords: Autism, screening tests, factor analysis, factor structure, latent structure.

\section{INTRODUCTION}

Autism is a range of neurodevelopmental disorders characterized by profound social impairment, impaired communication abilities and repetitive and stereotyped behavior according to DSM-5. The symptoms of autistic conditions are distributed on a scale of severity ranging from severe impairment in childhood autism to milder impairment in high-functioning autism and Asperger syndrome (ICD-10). An important question in research on autism is the underlying latent structure of autistic symptomatology and the extent to which autistic behavioural markers are interrelated. Recently it has been suggested that autistic conditions are dimensional with upper quantitative extremes of some of the traits, and it has further been implied that these traits are distributed continuously in the population [1-3]. Of interest for both researchers and practitioners is the psychometric structure of tools used for diagnosing and screening for autism, and the extent to which they reveal any dimensionality.

The Childhood Autism Spectrum Test (CAST) was originally developed as a tool designed to screen for high-functioning autism conditions in children [4]. It is a parental questionnaire comprising 37 items, with 31

*Address correspondence to this author at the Language Acquisition and Language Processing Lab, Norwegian University of Science \& Technology, Trondheim, Norway; Tel: +4773596791; E-mail: mila.vulchanova@ntnu.no key items targeting autism symptoms according to ICD10 (World Health Organization, 1993) and the DSM-IV (American Psychiatric Association, 1994). Its original English version was originally designed for identifying milder manifestations of autism (i. e. Asperger syndrome), but was subsequently found to also identify more severe autism i. e. childhood autism and has proven a reliable screening tool $[5,6]$. A moderate to good test-retest reliability has also been demonstrated in a sample enriched by high scorers on the CAST [6]. The CAST has been translated and adapted into over sixteen languages and cultures, which include German, Greek, Mandarin Chinese, Norwegian, Spanish, and Russian. Currently only one study reports its reliability in an adapted version. The study by Sun et al. [7] is based on a sample of 150 children aged 4-11 and compares the validity of the CAST to the Clancy Autism Behaviour Scale (CABS), a popular tool previously used in China. This study documents better validity for the CAST compared to the CABS, specifically in terms of sensitivity in distinguishing children with autism from children without autism.

The underlying structure of the symptoms that characterize autism is of interest both for cognitive accounts of what systems are compromised in autism, as well as for clinical purposes and achieving better diagnostic tools, and potentially, also in the search for genetic models of the etiology of the condition. A number of possibilities have been suggested, ranging 
from 4- and 5-dimensional models (based on ADI-R and ADOS, respectively $[8,9])$ to 1 -dimensional models, where degree of severity is crucial as measured on scores for the Social Responsiveness Scale (SRS) [10].

Of special interest for the current study are 3dimensional models in line with the original 3 categories included in DSM-IV: (1) Social interaction, (2) Communication and (3) Repetitive and stereotyped behaviours compared to a 2-dimensional model, where Social interaction and Communication are merged into a single factor, and are distinct from Repetitive and restricted behaviours and activities, reflecting the current diagnostic system, as prescribed in the DSM-5. The diagnostic criteria are divided into two large categories in this new version of the APA manual: (1) Deficits in social communication and social interaction across multiple contexts and (2) Restricted, repetitive patterns of behaviour, including verbal behaviour.

The development of models for the diagnosis of autistic spectrum disorders aims to meet expectations of a more detailed description of this heterogeneous group with the inclusion of categories that are less valid for other disorders. Besides the change from a multicategorial system to a single diagnostic category, an important characteristic of DSM-5, compared to the DSM-IV, is the presentation of the symptoms of ASD in a two-domain model, where the Communication deficits are combined with Social impairments in the group of social-communication deficits, while the Restricted and repetitive interests and behaviors differentiate the second group of symptoms [11, 12].

Two recent studies have addressed the factor structure of autistic symptoms in highly homogenous samples in early infancy. Beuker et al. [13] analyzed data from a sample of 11332 18-month-old infants from the Norwegian Mother and child cohort study (MoBA). A Confirmatory factor analysis (CFA) based on 44 items compiled from a number of sources (Early Screening of Autistic Traits (ESAT), the Modified Checklist for Autism in Toddlers (M-CHAT), Ages and Stages Questionnaire (ASQ)) revealed a 3-factor model, which displayed a significantly better fit than a 2 -factor model of the symptoms. Still, both the 2-factor and the 3-factor models have similar characteristics. Moreover, in the 3-factor model, there is a much higher correlation between the Social Interaction and Communication factors than the correlation between these two factors and the Repetitive behaviours factor. Importantly, the authors point out that symptoms that belong in the Social Interaction and Communication domains may have a different developmental pathway and manifestation in early development from symptoms that belong in the Stereotyped and rigid patterns of behaviour domain. The study by Guthrie et al., [14] explicitly compared the DSM-IV three criteria model and the DSM-5 two criteria model and their factor structure in a homogenous sample of 237 toddlers between 12 and 30 months of age. Their analysis demonstrates best fit for the 2-factor model, thus providing support for the DSM-5 model.

\section{CHILDHOOD AUTISM SPECTRUM TEST (CAST)}

The items included in the CAST do not presuppose an inherent structure. Neither are responses scored in terms of one, with cut-off points determined by an overall score which is the sum of points collected from ASD relevant responses. As such, the tool displays a one-dimensional structure. Still, it should be borne in mind that all included items target classical symptoms characterizing autism conditions (e. g., according to ICD-10 and DSM-IV), and, as such, the content of the scale adequately reflects all possible dimensions of the spectrum. The study by Sun et al. [1] is the first to address the latent structure and psychometric properties of CAST, albeit it's Mandarin translation. Based on a sample of 737 children from mainstream schools and 50 autistic cases, a categorical data factor analysis and Item Response Theory, this study found a two-factor best fit model for 28 out of the 31 keyed items included in CAST. The two factors were, respectively, Social and communication behavior, and Stereotyped and repetitive language and behaviour. The study found a moderate correlation between the two factors. The current study is the second study explicitly addressing the possible underlying structure of CAST. Our approach is mainly exploratory and attempts to reveal a possible dimensionality by assessing the adequacy of a range of factor models.

The above studies and the current one contribute to the discussion, and raised concerns of the appropriateness of the recently released DSM-5 diagnostic criteria for autism spectrum disorders (ASD) [15].

Revealing the latent structure of a test, considered as a set of items, is a key stage in its construction, especially in the estimation of its reliability and construct validity, and its standardization. This is a "critical issue" in the modeling of the raw test data, whether or not we assume a one-dimensional or a 
multidimensional model $[16,17]$. Unfortunately, the authors involved in the development of CAST, do not report data on the factor structure of the original version [4-6, 18-20]. The test appears to be, implicitly, considered as one-dimensional, which means that all test items refer to the same latent variable.

The current paper reports the first results of the study of the psychometric properties of the Bulgarian adaptation of the CAST [21]. Our main aim was to explore the extent to which the CAST can be used for screening for autism spectrum conditions (ASC) in children in Bulgaria. A specific objective of the first part of the study was to explore the underlying structure of autistic traits as measured by the CAST. This goal is especially relevant, with the recent publication of DSM5 , which, in contrast to DSM-IV, deconstructs autism symptoms into two distinct categories. Thus, the question arises whether collapsing Social interaction and Communication into a single criterion, in comparison to DSM-IV, reflects the dimensionality of autistic traits more accurately or not.

\section{METHODOLOGY}

\section{Participants}

The participants included 612 children (age range: 3-18 years), including children diagnosed with autistic spectrum disorder (ASD, $n=61$ ), and 551 children without any disorder (316 boys, and 296 girls). The participants were randomly selected in different age groups from six schools and three kindergartens in Sofia. The age groups are distributed as follows: The group of norms: Age 3-5 years $(n=56), 6-8$ years $(n=$ $167), 9-11$ years $(n=151), 12-14$ years $(n=101)$, and $15-18$ years $(n=76)$. The children with autism were diagnosed according to the criteria of ICD-10 by a certified clinician, and were sampled randomly from different age groups in the Program for children with autistic spectrum disorders in a day care center. The ASD group comprised: Age 3-5 years $(n=9), 6-8$ years ( $n=23), 9-11$ years $(n=17), 12-14$ years $(n=10)$ and $15-18$ years $(n=2)$. The original UK version of the CAST was only validated up to age 11 years. Our aim by including adolescent participants was to check the validity of the questionnaire for older participants in the absence of any screening tools in the Bulgarian context. The parents of all children received and filled out the Bulgarian adaptation of the CAST questionnaire. The Bulgarian CAST was developed in 2012 in a study which tested the adequacy of the translated CAST items in a group of native speaker parents and care-givers in Bulgaria [21]. The equivalence of the translation to the UK original was checked by back-translation by a professional. Written informed consent was obtained from the parents of all participants prior to the study.

\section{Factor Analysis of the Latent Structure of the Test}

In exploring the dimensionality of the latent structure of a measurement instrument, one can use a variety of approaches, methods and related indicators. Most of them are based on the idea of seeking some pattern of correlation between the manifested variables to be explained by the influence of one or more latent variables.

Factor analysis is an appropriate statistical technique for detecting latent structures and has been used for this purpose in different ASD diagnosing and screening tools, The Autism Diagnostic InterviewRevised (ADI-R), the Social Responsiveness Scale (SRS), CAST [1, 3, 13, 22].

Exploratory Factor analysis (EFA) is regarded primarily as a procedure for generating theoretical models and also as a method suitable for the development of psychological scales [23]. EFA is particularly suitable for exploring the latent structure of CAST, since its application aims to set up a factor model to explain the relationship among the observed variables. Confirmatory factor analysis (CFA) is treated as an extension of the "standard" exploratory factor analysis, and is therefore carried out under clear theoretical or empirical foundations, and its aim is the verification of a specific factor model. Specific hypotheses about the dimensionality of the factor space, the structure of the factor loadings of the variables and the correlations between them can be tested in this way [24, 25].

A main study in two phases was planned and completed to explore the factor structure of the Bulgarian adaptation of CAST. During the first phase we carried out an exploratory factor analysis (EFA) to generate a possible data model, and during the second - a confirmatory factor analysis (CFA) to verify this model. In order to avoid the effect of artificially increasing the extent of correspondence between the confirmatory model and the data, the full sample was divided randomly into two sub-samples with an equal size $\left(n_{1}=306\right.$ and $\left.n_{2}=306\right)$. The two types of factor analyses were carried out successively on these data 
sets - each type of analysis on one of the two data sets. Two tests - KMO and Bartlett's test of sphericity, were conducted as a preliminary step to check if the data from the first set could be efficiently factoranalyzed.

Principal axis factoring was applied during the first stage of the study as a method of building the model of the latent structure. In PFA, (unlike Principal Components Analysis (PCA), only a common, shared component of the variance of the manifested variables is used, which is due to common factors. Therefore, the extracted factors can be identified and conceptualized as psychological dimensions. A matrix of tetrachoric correlations of 31 scored items was used as the basis of the factor analysis. Tetrachoric correlations are suitable for the analysis of dichotomous variables, such as the items on the CAST, rather than Pearson $r$ (which are applicable for interval scales) or $\phi$ (for discrete binary scale). This approach to factorization of binary variables has been recommended in many studies [2630].

Two methods corresponding to the specificity of the PFA were applied to solve the problem of determining the optimal number of factors to be retained for rotation and included in the model - the popular graphical scree-test of Cattell [27, 31, 32] and a less popular, but more accurate parallel analysis (PA), developed by Horn [32-34].

According to Cattell's method, in order to determine the optimum number of factors to retain, one should find the point where the smooth decrease of eigenvalues appears to level off to the right of the plot. In this analysis we took a slightly different approach finding the point to the left of the break point, while not including that break point in the factor model [35].

The latent models developed at the exploratory stage of the study, were subjected to verification at the confirmatory stage by three groups of statistics: basic statistics (in particular, discrepancy function and RMS standardized residual); single sample fit indices (Joreskog GFI and AGFI, Bentler-Bonett normed fit index), and a group of indices based on the population non-centrality parameter (Steiger-Lind RMSEA index, McDonald non-centrality index, Population gamma index, etc.) The critical values of the indices showing high degree of model-data fit are according to Steiger [36].

\section{RESULTS}

\section{The Building of the Factor Model by Exploratory Factor Analysis (EFA)}

The Kaiser-Meyer-Olkin test of sampling adequacy was 0.861 , and the results of Bartlett's test of sphericity were $\chi^{2}(465)=3287.25 ; p=0.00$. The observed values of the two statistics are clear evidence that there are correlations between the test items that can be explained by the factor models.

The number of initially extracted factors in the unrotated factor solution was 26 . The eigenvalue of the first factor was 13.67 , explaining $44.10 \%$ of the total variance. The subsequent five factors had eigenvalues above 1.00 , respectively, $2.87(9.27 \%) 1.66(5.34 \%)$ $1.41(4.55 \%) 1.15(3.71 \%)$ and 1.03 (3.32\%). The cumulative proportion of the total variance, which can be explained by this group of 6 factors, was $70.29 \%$. The remaining factors had eigenvalues below 1.00 and explain smaller and smaller proportions of the total variance. A graph of the eigenvalues is represented in Figure 1.

Examining the unrotated factor solution, a strong, dominant first factor that accounted for nearly half of the total variance was observed. The ratio between its eigenvalue and that of the second factor is $\lambda_{F 1}: \lambda_{F 2}=$ 4.76, and between the second and the third factor $\lambda_{F 2}: \lambda_{F 3}=1.73$. This result is sufficient to suggest the existence of a one-dimensional latent space with a major (first) factor [17, 28, 37-39].

As shown in Figure 1, Cattell's graphical scree-test suggests a 2-factor solution. The application of the 2factor model with orthogonal varimax normalized rotation of factors leads to the separation of two relatively equal factors with explained variance of 8.24 and 7.42 , respectively. The Varimax normalized method was performed not only because it is most commonly used, but also because it yielded a better pattern of loadings. Distributed between the factors according to their factor loadings, 15 items can be assigned to the first factor, and 16 , to the second. The value of 0.40 was considered as the lower borderline of acceptability, in order for an item to be used in the interpretation of the respective factor [40].

Among the items, which correlate highly with the first factor, are those with numbers 27. Does s/he make normal eye-contact? (factor loading 0.78 ); 10 . Does s/he find it easy to interact with other children? (0.76); 13. Does s/he mostly have the same interests as 


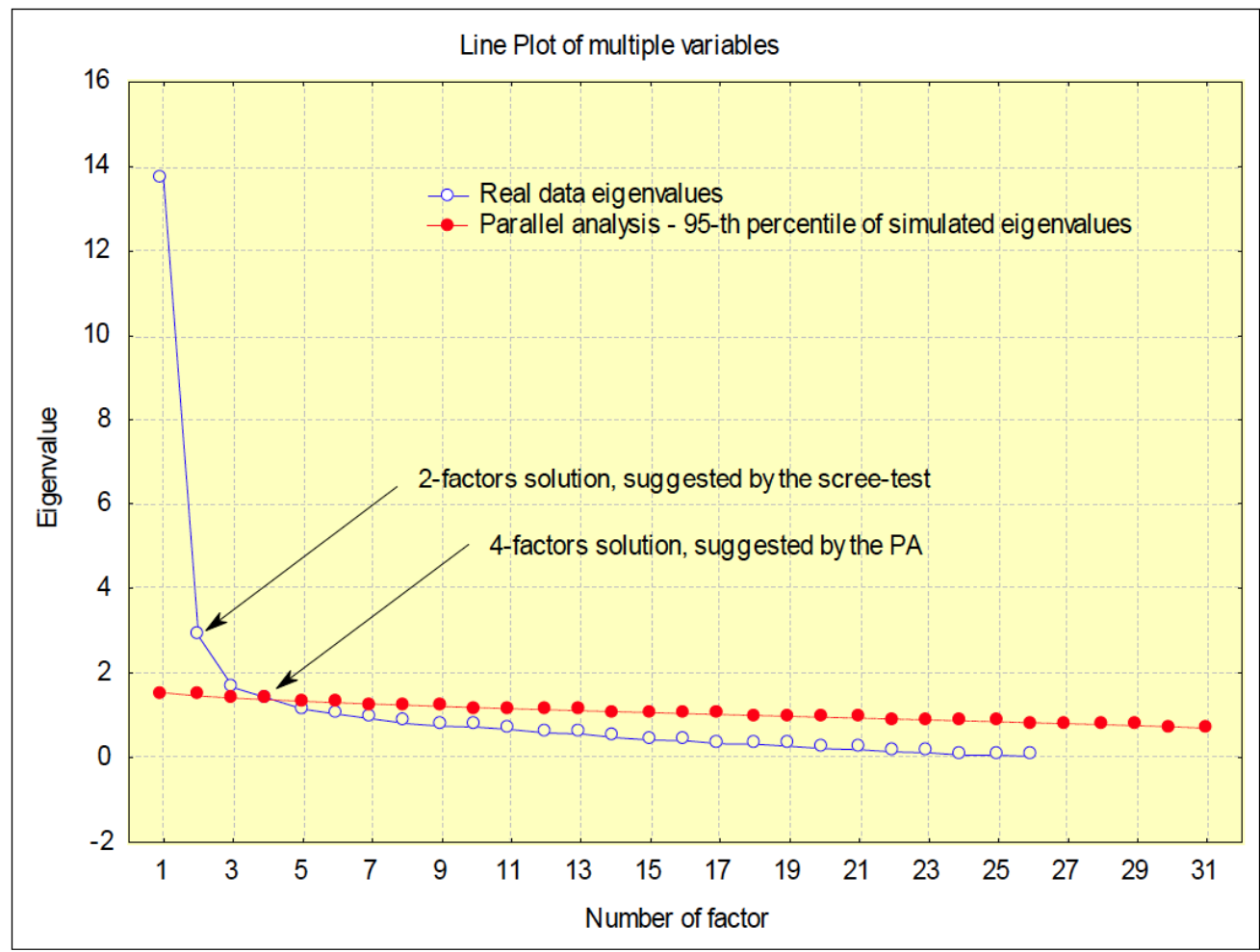

Figure 1: Real and simulated eigenvalues.

his/her peers? (0.76); and others. These items reflect aspects of communication and the relationship of the child with other children/individuals. Among the items that correlate highly with the second factor are 28. Does s/he have any unusual and repetitive movements? (factor loading 0.71); 37 . Does s/he have odd or unusual phrases? (0.71); 9. Does s/he like to do things over and over again, in the same way all the time? (0.70); and others. The items from this group reflect individual characteristic features, such as attention to detail, repetition of actions, concentration and unilateralism in the child's behaviour.

The factor loadings of most of the items on the two factors are characterized by predominantly positive, moreover, very high values. Only 3 items have slightly negative values on the first (numbers 6, 7 and 19) and respectively on the second factor $(8,16$ and 31$)$, and thus cannot be considered as cross-loading items. Out of 15 items in the first factor, 13 items have loadings greater than 0.40 . Regarding the second factor, factor loadings $>0.40$ were observed for all 16 items.

The items of factor 1 from the two-factor model, reflecting aspects of communication and the relationship of the child with other children, were divided, albeit unevenly, in the "new" factors 1 and 3. Almost all the items from the "former" factor 1 , with numbers 10. (factor loading 0.84), 27. (0.82), 13. (0.79), 17. (0.78), 1 (0.77), 11 (0.71) and others can be assigned to factor 1 in the 4 -factor model. But its interpretation is hampered by the presence of three items from the "former" factor 2 that have sufficiently large factor loadings, namely item 18. Does s/he have difficulty understanding the rules for polite behavior? (factor loading 0.60), 28. Does s/he have any unusual and repetitive movements? (0.72) and 29. Is his/her social behaviour very one-sided and always on his/her own terms? (0.42). Items 18. and 29. reflect some problems in the child's behavior in general, especially observable in the younger age group, and are not particularly problems of social communication. Item 28 . refers to repetitive behaviours. Factor 3 in the 4-factor model contains only two items, both of which were highly specific. These are item 8 . When s/he was 3 years old, did s/he spend a lot of time pretending (e.g., play-acting being a superhero, or holding teddy's tea parties? (factor loading 0.89) and 31. Does s/he prefer imaginative activities such as play-acting or storytelling, rather than numbers or lists of facts? (0.53). Both items reflect the tendency of the child to use imagination and role-play. Alternatively, this result may be simply due to the similar wording of the questions. The removal of items measuring imaginative play substantially improved the 2-factor model in a recent study by Mandy et al. [41]. 


\section{Verification of the Two Models by Confirmatory Factor Analysis}

The two factor models identified in the previous stage of the analysis were subjected to a sequential confirmatory factor analysis under the assumption that the factors in the respective model are uncorrelated (orthogonal). The method of estimation used is Generalized Least Squares followed by Maximum Likelihood (GLS - ML). The results of these analyzes are presented in columns 3 and 5 in Table 1. The two sets of statistical indicators suggest consistently, that the hypotheses of adequacy of the two- or four-factor orthogonal models of the underlying structure of CAST should be rejected.
A disturbing fact are the large factor loadings of some items on the unique factors, which even exceed those for the common factors. This means that a larger part of the total variance in these items is due to the influence of the unique, and not the common factors. This, in turn, can be considered as a signal that the tested models are not adequate to the actual data.

Furthermore, the observed values of discrepancy functions, which are a family of functions used to check the extent to which the tested model is consistent with the empirical data, are 13.82 for the 2-factor, and 13.75 for the 4-factor model. This is evidence that none of them represents a good description of the latent structure of CAST. The observed values of the Root

Table 1: Test Statistics of the Confirmatory Factor Analysis with Orthogonal and Oblique Factors

\begin{tabular}{|c|c|c|c|c|c|c|c|}
\hline \multirow{2}{*}{ Statistics/ test } & \multirow{2}{*}{\multicolumn{2}{|c|}{$\begin{array}{c}\text { Critical/ } \\
\text { acceptable value }\end{array}$}} & \multicolumn{3}{|c|}{ 2-factor model } & \multicolumn{2}{|c|}{ 4-factor model } \\
\hline & & & \multicolumn{2}{|c|}{ uncorrelated factors } & correlated factors & uncorrelated factors & correlated factors \\
\hline 1 & \multicolumn{2}{|c|}{2} & \multicolumn{2}{|r|}{3} & 4 & 5 & 6 \\
\hline Discrepancy Function & & & \multicolumn{2}{|r|}{13.824} & 12.564 & 13.750 & 12.105 \\
\hline ICSF Criterion & & & \multicolumn{2}{|r|}{0.000} & 0.000 & 0.000 & 0.000 \\
\hline ICS Criterion & & & \multicolumn{2}{|r|}{0.000} & 0.000 & 0.000 & 0.000 \\
\hline ML $\chi 2$ & & & \multicolumn{2}{|c|}{8446.621} & 7676.849 & 8401.423 & 7395.892 \\
\hline Df & \multicolumn{2}{|c|}{-} & \multicolumn{2}{|r|}{434} & 433 & 435 & 428 \\
\hline$\alpha$ & \multicolumn{2}{|c|}{0.05} & \multicolumn{2}{|r|}{0.000} & 0.000 & 0.000 & 0.000 \\
\hline RMS Standardized Residual & & & \multicolumn{2}{|r|}{0.283} & 0.100 & 0.288 & 0.097 \\
\hline \multicolumn{8}{|c|}{ Single sample fit indices } \\
\hline Joreskog GFI & \multicolumn{2}{|r|}{$>0.95$} & & 0.549 & 0.545 & 0.561 & 0.573 \\
\hline Joreskog AGFI & \multicolumn{2}{|r|}{$>0.95$} & & 0.485 & 0.479 & 0.499 & 0.505 \\
\hline $\begin{array}{l}\text { Bentler-Bonett Normed Fit } \\
\text { Index }\end{array}$ & \multicolumn{2}{|r|}{$\cong 1.00$} & & 0.556 & 0.596 & 0.558 & 0.611 \\
\hline \multicolumn{8}{|c|}{ Population noncentrality parameter } \\
\hline $\begin{array}{c}\text { Population Noncentrality } \\
\text { Parameter }\end{array}$ & \multicolumn{3}{|c|}{$\begin{array}{c}\text { lower } 90 \% \\
\text { point } \\
\text { upper } 90 \%\end{array}$} & \begin{tabular}{l|}
11.561 \\
12.023 \\
12.498
\end{tabular} & $\begin{array}{l}11.762 \\
12.228 \\
12.706\end{array}$ & $\begin{array}{l}10.968 \\
11.419 \\
11.881\end{array}$ & $\begin{array}{l}10.408 \\
10.847 \\
11.299\end{array}$ \\
\hline Steiger-Lind RMSEA Index & $<0.05$ & \multicolumn{2}{|c|}{$\begin{array}{l}\text { lower } 90 \% \\
\text { point } \\
\text { upper } 90 \%\end{array}$} & $\begin{array}{l}0.163 \\
0.166 \\
0.170\end{array}$ & $\begin{array}{l}0.165 \\
0.168 \\
0.171\end{array}$ & $\begin{array}{l}0.159 \\
0.162 \\
0.165\end{array}$ & $\begin{array}{l}0.156 \\
0.159 \\
0.162\end{array}$ \\
\hline $\begin{array}{l}\text { McDonald Noncentrality } \\
\text { Index }\end{array}$ & $>0.95$ & \multicolumn{2}{|c|}{$\begin{array}{l}\text { lower } 90 \% \\
\text { point } \\
\text { upper } 90 \%\end{array}$} & $\begin{array}{l}0.002 \\
0.002 \\
0.003\end{array}$ & $\begin{array}{l}0.002 \\
0.002 \\
0.003\end{array}$ & $\begin{array}{l}0.003 \\
0.003 \\
0.004\end{array}$ & $\begin{array}{l}0.004 \\
0.004 \\
0.005\end{array}$ \\
\hline Population Gamma Index & $>0.95$ & \multicolumn{2}{|c|}{$\begin{array}{l}\text { lower } 90 \% \\
\text { point } \\
\text { upper } 90 \%\end{array}$} & $\begin{array}{l}0.554 \\
0.563 \\
0.573\end{array}$ & $\begin{array}{l}0.550 \\
0.559 \\
0.569\end{array}$ & $\begin{array}{l}0.566 \\
0.576 \\
0.586\end{array}$ & $\begin{array}{l}0.578 \\
0.588 \\
0.598\end{array}$ \\
\hline $\begin{array}{l}\text { Adjusted Population } \\
\text { Gamma Index }\end{array}$ & $>0.95$ & \multicolumn{2}{|c|}{$\begin{array}{l}\text { lower } 90 \% \\
\text { point } \\
\text { upper } 90 \%\end{array}$} & $\begin{array}{l}0.490 \\
0.501 \\
0.512\end{array}$ & $\begin{array}{l}0.484 \\
0.495 \\
0.506\end{array}$ & $\begin{array}{l}0.505 \\
0.516 \\
0.528\end{array}$ & $\begin{array}{l}0.511 \\
0.523 \\
0.534\end{array}$ \\
\hline
\end{tabular}


mean square (RMS) criterion (0.283 and 0.288$)$ are above the critical value of 0.05 and unacceptable from the point of view of the goodness-of-fit of the models. The test for general adequacy $\chi^{2}$ of the inspected model (Table 1 ) shows extremely high values in both models $\left(\chi^{2}=8446.62, d f=434\right.$ for the 2-factor and $\chi^{2}=$ 8401.42 , $d f=435$ for the 4 r-factor model), and low level of associated probabilities $(p=0.00)$. Based on the results of these tests, the hypotheses of a 2-factor, and, respectively a 4-factor latent structure that generates shared variance of responses of the subjects, may be rejected at a significance level of $\alpha=$ 0.05 .

The observed values of other statistics, such as single sample fit indices (Goodness-of-fit index (GFI)), Adjusted goodness-of-fit index (AGFI), and BentlerBonett normed fit index were used to evaluate the adequacy of the tested models and lead to the same conclusion (Table 1).

A radical change in approach for evaluation of the adequacy of the model tested on this data set is based on the estimation of the population non-centrality parameter. Instead of testing the null hypothesis, the opposite approach is proposed - to establish to what extent it is inadequate, how far is the model from the population and how precisely can this inadequacy be determined based on the sample data. This leads to a similar result. Neither the point estimates of the indices, nor the values within the confidence intervals $(90 \%)$, give reason to assume that the two tested models are adequate to the data.

The comparison of the two series of statistics describing the adequacy of the two tested models with orthogonal factors, shows an expected trend - they are more favorable to the 4-factor than the 2-factor model, due to the complexity of the former and the simplicity of the latter model.

\section{Towards a Simple Factor Structure}

The conducted analyses and their results contain a number of indications that the inadequacy of the tested models could be due to the chosen strategy of orthogonal rotation of the factors. In a series of publications Thurstone [42-45] developed the concept of achieving a simple factor structure as the goal of factor analysis. Thurstone's conception regards the third and final phase of the factor analysis, at which the rotation of the factors of the chosen model is performed in order to reach the most simple, but theoretically meaningful model of relations between the manifested and latent variables. Thorndike further suggests that the maximum simplicity of the structure is achieved when a given variable has a definite (high) loading on only one of the factors, and zero loadings on all other factors [46]. On a less restrictive interpretation, this rule implies that a simple factor structure is obtained when a given manifested variable has large loading on one factor, and low loadings on the others.

In both models, many of the items that load on a given factor, display large loadings on that factor (above 0.40). Furthermore, we observe that in the 2factor model, over $60 \%$ of all items have such high factor loadings on each factor. Many of them correlate, to an approximately equal extent, with both factors, and serve as "bridges" between them. Some examples of such item cross-loadings are item 18. (with factor loadings on the first and the second factor, respectively 0.55 and 0.57 ), item 10. (0.76 and 0.54) item 28 (0.64 and 0.71 ), and others. The existence of such "bridges" makes the interpretation of the factors difficult. Moreover, this is also evidence of the existence of correlations between them.

The factor matrix of the 4-factor model contains more contrasting factor loadings of the individual items and provides more opportunities of a clear definition of their factor identity. Nevertheless, there are many items with comparable factor loadings on at least two factors. Such cross-loading items are 9. (factor loading of 0.46 on factor $1,0.54$ on factor $2,-0.24$ on factor 3 and 0.33 on factor 4 ), item 15 . (0.57 on factor 1 and 0.46 on factor 4 ), item 28. (0.72 on factor 1 and 0.56 on factor 4 ) and others. The results of the analyses so far lead to the conclusion that the orthogonal rotation of the factors does not ensure a simple factor structure. It seems that the latent structure is composed of correlated, non-orthogonal (oblique) factors.

As a result, we conducted a second iteration of the confirmatory factor analysis to verify the hypotheses of a two- and a four-factor model of the data with correlated factors. The results are presented in Table 1 , columns 4 and 6 . The statistics of the models with non-orthogonal factors, however, are comparable to those of the models with orthogonal factors. Thus, these two latter models with correlated factors also fail in uncovering the latent structure of the data.

\section{Hierarchical Factor Model}

Hierarchical factor analysis is based on a type of rotation of the factor space, which "allows" the factors 
to correlate. The concept of non-orthogonal (oblique) factors is often exploited in research in the social sciences and humanities, based on the expectation that the latent dimensions are not isolated from each other. In such cases, the non-orthogonal rotation could result in a more accurate, and a more reproducible factor structure $[35,47]$.

We applied a hierarchical factor analysis at this third step of analysis as a development of the "conventional" methodology of rotation of the oblique factors. The factors are rotated, in order to present the clusters of variables in the best way, without constraints on orthogonality. The correlation matrix of the oblique factors is then submitted to secondary factorization to extract the final structure of the orthogonal factors. It divides the variability of the observed variables into two parts: a variance which is due to the common (secondary) factors, and a unique variance which is attributable to the clusters of variables (primary factors).

The hierarchical factor analysis was conducted with the restrictive condition that the minimum eigenvalue of the extracted factors should be 1.33. The rationale was that this was the lower threshold level, corresponding to the 95th percentile of the distribution of the eigenvalues of the fourth random factor, extracted by the method of Parallel analysis. As a result of this procedure, two primary factors, and one secondary factor were extracted.

We found high correlations between the two variable clusters (oblique factors) and the secondary factor (for each cluster $r=0.84$ ). The correlations of the first cluster with primary 1 and primary 2 are, respectively 0.55 , and 0.00 , while these of the second cluster with primary 1 and primary 2 are respectively, 0.00 and 0.55 . The correlation between the two oblique factors was also high $(r=0.70)$, which suggests that the applied method of rotation is adequate.

The two clusters of items have approximately the same size. The first cluster, which determines primary 1, includes 15 items from the test. After orthogonal varimax normalized rotation (see Table 2), the factor loadings of 13 of these items were higher than 0.40 , and 7 of them have factor loadings that exceed 0.70 . The items with factor loadings less than 0.40 are number 8. (factor loading 0.32) and 31. (0.29). All the items in this cluster relate to one particular area deficits in social communication and social interaction. The second cluster that determines primary 2 , is formed of 14 items, each of which has a factor loading higher than 0.40 , and also there are three items with factor loading greater than 0.70 . Most of the items in this cluster are associated with another specific area restricted, repetitive patterns of behaviour.

In this model, there were only two items with large, almost identical cross-loadings on the two primary factors. They were item 18. Does s/he have difficulty understanding the rules for polite behaviour? ( 0.55 and 0.57 ) and 34 . Does s/he try to impose routines on him/herself, or on others, in such a way that it causes problems?, (0.45 and 0.46). Therefore, formally these items cannot be assigned to either of the two primary

Table 2: Factor Loadings on Secondary and Primary Factors in the Hierarchical Model

\begin{tabular}{|c|c|c|c|}
\hline \multirow{2}{*}{ Item No. } & \multicolumn{3}{|c|}{ Factors } \\
\hline & Secondary & Primary 1 & Primary 2 \\
\hline 1 & 0.74 & ${ }^{*} 0.71$ & 0.44 \\
\hline 2 & 0.46 & ${ }^{*} 0.65$ & 0.07 \\
\hline 5 & 0.60 & ${ }^{*} 0.64$ & 0.29 \\
\hline 6 & 0.21 & -0.16 & ${ }^{*} 0.49$ \\
\hline 7 & 0.35 & -0.00 & ${ }^{*} 0.54$ \\
\hline 8 & 0.08 & ${ }^{*} 0.32$ & -0.18 \\
\hline 9 & 0.61 & 0.25 & ${ }^{*} 0.70$ \\
\hline 10 & 0.84 & ${ }^{*} 0.76$ & 0.55 \\
\hline 11 & 0.74 & ${ }^{*} 0.72$ & 0.43 \\
\hline 13 & 0.78 & ${ }^{*} 0.76$ & 0.45 \\
\hline 14 & 0.48 & 0.20 & ${ }^{*} 0.55$ \\
\hline 15 & 0.68 & ${ }^{*} 0.57$ & 0.49 \\
\hline 16 & 0.31 & ${ }^{*} 0.70$ & -0.21 \\
\hline 17 & 0.69 & ${ }^{*} 0.73$ & 0.35 \\
\hline 18 & 0.72 & 0.55 & 0.57 \\
\hline 19 & 0.20 & -0.14 & ${ }^{*} 0.46$ \\
\hline 20 & 0.73 & 0.46 & ${ }^{*} 0.69$ \\
\hline 21 & 0.51 & ${ }^{*} 0.53$ & 0.26 \\
\hline 23 & 0.59 & 0.35 & ${ }^{*} 0.57$ \\
\hline 24 & 0.57 & ${ }^{*} 0.61$ & 0.28 \\
\hline 25 & 0.59 & 0.26 & ${ }^{*} 0.67$ \\
\hline 27 & 0.73 & ${ }^{*} 0.78$ & 0.36 \\
\hline 28 & 0.87 & 0.64 & ${ }^{*} 0.72$ \\
\hline 29 & 0.52 & 0.32 & ${ }^{*} 0.49$ \\
\hline 30 & 0.68 & 0.44 & ${ }^{*} 0.63$ \\
\hline 31 & 0.15 & ${ }^{*} 0.29$ & -0.06 \\
\hline 32 & 0.56 & 0.30 & ${ }^{*} 0.57$ \\
\hline 34 & 0.59 & 0.45 & 0.46 \\
\hline 35 & 0.61 & ${ }^{*} 0.66$ & 0.30 \\
\hline 36 & 0.47 & 0.14 & ${ }^{*} 0.59$ \\
\hline 37 & 0.68 & 0.36 & ${ }^{*} 0.71$ \\
\hline
\end{tabular}

${ }^{*}$ Clusters of loadings are marked with asterisks. The clusters determine the two oblique factors. 
factors. The decision for their factor assignment has been taken on conceptual grounds, based on their semantic similarity with the items in the respective cluster of descriptors of autism spectrum disorder.

Thus, primary 1 is formed by the following 16 items with numbers $1,2,5,8,10,11,13,15,16,17,18,21$, $24,27,31$ and 35 . The first factor is marked by large factor loadings of items 27 . Does s/he make normal eye-contact? (factor loading 0.78 ), 10. Does s/he find it easy to interact with other children? (0.76), 13. Does s/he mostly have the same interests as his/her peers? (0.76), 11. Can s/he keep a two-way conversation going? (0.72) and others. The assignment of item 13 to primary 1 can be questioned against the other items in that cluster, since responses to this item can be driven by ambivalent considerations. On the one hand, the interpretation may be that the interests of the child in relation to those of his/her peers are being evaluated by the parents as causing problems in social interaction. Alternatively, an interpretation can be as indication of stereotyped behaviors and repetitive movements, if the activities of the child are evaluated from the point of view of his/her rituals and desire to preserve sameness and own routines.

Primary 2 includes the following 15 items with numbers $6,7,9,14,19,20,23,25,28,29,30,32,34$, 36 and 37 . The highest loadings are for item 28. Does s/he have any unusual and repetitive movements? (factor loading 0.72), 37. Does s/he have odd or unusual phrases? (0.71), 9. Does s/he like to do things over and over again, in the same way all the time? (0.70), 20. Is his/her voice unusual (e.g., overly adult, flat, or very monotonous)? (0.69) Two items could be considered ambivalent, 29. Is his/her social behaviour very one-sided and always on his/her own terms? and 36. Does s/he often turn conversations to his/her favorite subject rather than following what the other person wants to talk about? A possible interpretation here can be that responses to these items reflect the parents' view of the behaviour leading to two perspectives - from the point of view of social rules or with a focus on permanence and lack of flexibility.

On the basis of these results, it is not difficult to identify primary 1 as "Deficits in social communication and social interaction", a primary 2 - as "Restricted, repetitive patterns of behavior".

A look at the items in the common second-order factor reveals that the two clusters of items are presented in it in a balanced way. 25 of the 31 items have factor loadings higher than 0.40 , and 8 out of those have factor loadings higher than 0.70. Among the items that are representative of the common factor (i. e., the items with the highest factor loadings), we find representatives of the group reflecting deficits in social communication and social interaction (primary 1) and the group representing restricted, repetitive patterns of behaviour (primary 2). Among them are item 28. Does s/he have any unusual and repetitive movements? (factor loading 0.87 ), 10. Does s/he find it easy to interact with other children? (0.84), 13. Does s/he mostly have the same interests as his/her peers? (0.78), and others.

It is interesting that the items with the lowest factor loadings on the common factor are those which refer to difficulties in imaginative play. These are two items with numbers 8 . When s/he was 3 years old, did s/he spend a lot of time pretending (e.g., play-acting being a superhero, or holding teddy's tea parties)? (0.08), and 31. Does s/he prefer imaginative activities such as play-acting or story-telling, rather than numbers or lists of facts? (0.15). These items load on the factor "Deficits in social communication and social interaction", and are characterized by relatively low loadings on that factor (respectively, 0.32 and 0.29 ). We could recall also that in the 4-factor model with orthogonal factors, exactly (and only) these two items formed one of the separate factors.

\section{DISCUSSION}

The present study sought to achieve one main goal - revealing the factor (scale) structure of the Bulgarian adaptation of CAST. The psychometric properties of screening tools of this type are of interest for both practitioners and researchers from the point of view of how well they reflect clusters of symptoms included in the diagnosis and the autism phenotype. This information is also a prerequisite for further developments in the direction of adapting and, ultimately, standardizing the test in the Bulgarian cultural context.

A two-step procedure for generating a factor model through exploratory factor analysis, and its verification by confirmatory factor analysis was undertaken for the detection of features in the Bulgarian adaptation of CAST. These methods led to the constitution of two, seemingly mutually exclusive, factor models - one with 2 , and one with 4 orthogonal factors.

Scott et al. [4] note that the items in CAST (originally Childhood Asperger Syndrome Test) as manifested variables include different descriptions of behaviors according to ICD-10 and DSM-IV, which 
reflect the main characteristics of the autism spectrum disorder. In particular, these characteristics in the DSM-IV are divided into three categories: (1) Impairment in social interaction, (2) Impairments in communication and (3) Restricted, repetitive, or stereotyped patterns of behaviour, interests, or activities. Some of the items in CAST are based on the items from two other screening tests - the Pervasive Developmental Disorders Questionnaire (PDD-Q) and the Asperger Syndrome Screening Questionnaire (ASSQ) (ibid.) Despite these various sources, the authors have aimed to cover a wide range of behaviors in order to make the test sensitive in performing its screening function (ibid.) Therefore, it would be plausible to expect that the items in the test will be organized in a three-factor structure, reflecting the structure of the descriptions in DSM-IV.

The distribution of the items in the 2-factor model generally follows this structure. Almost all the items that refer to the characteristics of the first category (Impairment in social interaction), load on the first factor (except item 18.), and all the items from the third category (Restricted, repetitive, or stereotyped patterns of behaviour), with no exception - on the second. Particularly interesting is the distribution of the items which relate to the second category of characteristics in DSM-IV (Impairments in communication) - they are fairly evenly divided between the two factors. The items describing (impairment in) communication activities in social context, such as make-believe or social imitative play (items 8,24 and 31 ) or the ability to initiate or sustain a conversation (11), are assigned to the first factor. The items that reflect restricted or repetitive patterns in language and behavior, as idiosyncratic language (item 37 ), stereotyped use of language (item 36 ) and others, load on factor 2.

In the 4-factor model each of the above two factors split into two other factors. Factor 1 from the previous model is generally preserved, but a small group of items from the second category in DSM-IV (impairments in communication), expressing the (lack of) make-believe and social-imitative play (items 8 and 31), now form an independent factor. Interestingly, item 24 is not part of this new factor, despite its belonging to the same sub-category. The second factor also splits into two other factors, and each of them contains a mixture of items belonging to the second (impairments in communication) and the third (restricted, repetitive, or stereotyped patterns of behaviour) categories in DSM-IV. In one of them, there is a prevalence of items from the category "Restricted, repetitive, or stereotyped patterns of nonverbal behavior", and in the other items reflecting the same features in verbal behaviour and in social context.

The adequacy of these two factor models was not verified, also under the assumption of nonorthogonality of the dimensions. Despite the negative outcome, these preliminary analyses, however, led to an important conclusion. Firstly, they point to the apparent lack of coherence between the items referred to in the second category of behaviors described in DSM-IV. These items refer to two distinct groups of behaviours (e. g. communicative aspects of language and imaginative play) and, thus gravitate to different dimensions. Another finding is that some of the items in both models are influenced equally by different factors, making it difficult to differentiate them, even under the assumption that the factors are oblique. This highlights the necessity of another kind of rotational strategy, and searching for a hierarchical, instead of a 'flat' structure with primary factors. An evaluation of the lower threshold eigenvalue of simulated factors was carried out by Parallel analysis, where the extracted factors make both statistical and psychological "sense".

The structure, which was revealed by the analyses as adequate to the data, consists of two primary and one secondary (common) factor. These two primary dimensions, which are also orthogonal, basically reproduce the two-factor model described above and are consistent with the psychometric structure of the Mandarin Chinese CAST reported in Sun et al. [1]. From these results it becomes evident that the features of the autism spectrum, covered by the second category in DSM-IV, do not form a single coherent unit, as is the case with the characteristics of the other two categories. Indeed, part of the features in the category of "language and communication" are linked to the use of language as a means of (social) communication and interaction, rather than features of language competence per se, and can be considered as part of the first category (Impairment in social interaction). Another aspect of these features is linked to characteristics of speech at the individual level, such as stereotyped or repetitive and idiosyncratic speech, again not reflecting language competence as such, and can be assigned to the third category (Restricted, repetitive, or stereotyped patterns of behaviour). From this perspective, the factor structure at the primary level in the hierarchical analysis reproduces more accurately the structure of the diagnostic criteria, represented in the new DSM-5. The diagnostic criteria are divided into 
two categories in this new version of the APA manual: (1) Deficits in social communication and social interaction across multiple contexts and (2) Restricted, repetitive patterns of behaviour, including verbal behaviour. All the items in primary 1 can be attributed unambiguously to the first category, and in primary 2 to the second DSM-5 category. These two categories are presented in a balanced way in the common second-order factor.

This structure is consistent with the two main categories adopted in the DSM-V and current accounts of the range of conditions broadly falling into two main clusters of symptoms [48]. Our findings are also consistent with the results of the only other study so far, which has addressed the psychometric properties of the Mandarin Chinese CAST [1]. Unlike the latter study, however, we applied an "agnostic" approach subjecting the data to exploratory and confirmatory factor analyses, first assuming a flat, non-dimensional structure. Even though the questions in the CAST include the most common symptoms from the DSM-IV and ICD-10 criteria, the cut-off points are based on the sum of scores on ASD target responses, which does not presuppose any inherent underlying structure. Thus, our aim was to check whether any latent structure emerged from responses in our sample. We further demonstrate that the CAST can be used successfully in other languages and cultures for screening for autism. Unlike the original UK CAST, our study included a sample with an extended upper limit (up to 18 years). The results of the analyses indicate that the Bulgarian CAST can be used also for age groups not envisaged in the original UK version. This is especially relevant in the Bulgarian context where the number of the screening tools for autism is limited. Further cross-linguistic and cross-cultural studies may reveal the extent to which this is tenable in other contexts.

\section{REFERENCES}

[1] Sun X, Allison C, Auyeung B, Matthews FE, Norton S, BaronCohen S, Brayne C. Psychometric Properties of the Mandarin Version of the Childhood Autism Spectrum Test (CAST): An Exploratory Study. Journal of Autism and Developmental Disorders 2014a; 44(7): 1565-76. http://dx.doi.org/10.1007/s10803-013-2024-3

[2] Wing L. The autistic continuum. In: L. Wing, editor. Aspects of autism: Biological Research. Royal College of Psychiatrists, London: Gaskell 1988.

[3] Constantino JN, Todd RD. Autistic traits in the general population: A twin study. Archives of General Psychiatry 2003; 60(5): 524-530.

http://dx.doi.org/10.1001/archpsyc.60.5.524

[4] Scott FJ, Baron-Cohen S, Bolton P, Brayne C. The CAST (Childhood Asperger Syndrome Test): Preliminary development of a UK screen for mainstream primary-schoolage children. Autism 2002a; 6(1): 9-31.

http://dx.doi.org/10.1177/1362361302006001003

[5] Williams J, Scott F, Scott C, Allison C, Bolton P, BaronCohen S, Brayne C. The CAST (Childhood Asperger Syndrome Test): Test accuracy. Autism 2005; 9(1): 45-68. http://dx.doi.org/10.1177/1362361305049029

[6] Allison C, Williams J, Scott F, Stott C, Bolton P, Baron-Cohen $\mathrm{S}$, Brayne C. The Childhood Asperger Syndrome Test (CAST): Test-retest reliability in a high scoring sample. Autism 2007; 11(2): 173-185. http://dx.doi.org/10.1177/1362361307075710

[7] Sun X, Allison C, Auyeung B, Matthews FE, Zhang Z, BaronCohen S, Brayne C. Comparison between a Mandarin Chinese version of the Childhood Autism Spectrum Test and the Clancy Autism Behaviour Scale in mainland China. Research in Developmental Disabilities 2014b; 35: 15991608.

http://dx.doi.org/10.1016/j.ridd.2014.02.005

[8] Lord C, Rutter M, Le Couteur A. Autism Diagnostic Interview - Revised: a revised version of a diagnostic interview for caregivers of individuals with possible pervasive developmental disorders. Journal of Autism and Developmental Disorders 1994; 24: 659-685. http://dx.doi.org/10.1007/BF02172145

[9] Lord C, Risi S, Lambrecht L, Cook EH Jr, Leventhal BL, DiLavore PC, Pickles A, Rutter M. The autism diagnostic observation schedule-generic: a standard measure of social and communication deficits associated with the spectrum of autism. Journal of Autism and Developmental Disorders 2000; 30(3): 205-23.

http://dx.doi.org/10.1023/A:1005592401947

[10] Constantino JN, Przybeck T, Friesen D, Todd RD. Reciprocal social behavior in children with and without pervasive developmental disorders. Journal of Developmental and Behavioral Pediatrics 2000; 21: 2-11.

http://dx.doi.org/10.1097/00004703-200002000-00002

[11] Frazier TW, Youngstrom EA, Speer L, Embacher R, Law P, Constantino J, Findling RL, Hardan AY, Eng C. Validation of proposed DSM-5 criteria for autism spectrum disorder. Journal of the American Academy of Child and Adolescent Psychiatry 2012; 51: 28-40. http://dx.doi.org/10.1016/j.jaac.2011.09.021

[12] Grzadzinski R, Huerta M, Lord C. DSM-5 and autism spectrum disorders (ASDs): an opportunity for identifying ASD subtypes. Molecular Autism 2013; 4: 12. http://dx.doi.org/10.1186/2040-2392-4-12

[13] Beuker KT, Schjølberg S, Kveim LK, Donders R, Lappenschaar M, Swinkels SH, Buitelaar JK. The structure of autism spectrum disorder symptoms in the general population at 18 months. Journal of Autism and Developmental Disorders 2013; 43(1): 45-56. http://dx.doi.org/10.1007/s10803-012-1546-4

[14] Guthrie W, Swineford LB, Wetherby AM, Lord C. Comparison of DSM-IV and DSM-5 factor structure models for toddlers with autism spectrum disorder. Journal of the American Academy of Child and Adolescent Psychiatry 2013; 52: 797805.

http://dx.doi.org/10.1016/j.jaac.2013.05.004

[15] Wing L, Gould J, Gillberg C. Autism spectrum disorders in the DSM-V: better or worse than the DSM-IV? Developmental Disabilities Research Reviews 2011; 32: 76873.

http://dx.doi.org/10.1016/j.ridd.2010.11.003

[16] Embretson SE, Reise SP. Item response theory for psychologists. Lawrence Mahwah, NJ: Erlbaum Associates, Inc. 2000; p. 227. 
[17] De Ayala RJ. The theory and application of Item response theory. New York: Guilford Publishing; 2009.

[18] Scott FJ, Baron-Cohen S, Bolton P, Brayne C. Brief report: Prevalence of autism spectrum conditions in children aged 511 years in Cambridgeshire, UK. Autism 2002b; 6(3): 231237.

http://dx.doi.org/10.1177/1362361302006003002

[19] Williams J, Allison C, Scott F, Stott C, Bolton P, Baron-Cohen S, Brayne C. The Childhood Asperger Syndrome Test (CAST): Test-retest reliability. Autism 2006; 10(4): 415-427. http://dx.doi.org/10.1177/1362361306066612

[20] Williams JG, Allison C, Scott FJ, Bolton PF, Baron-Cohen S, Matthews FE, Brayne C. The Childhood Autism Spectrum Test (CAST): Sex differences. Autism 2008; 38(9): 1731-9. http://dx.doi.org/10.1007/s10803-008-0558-6

[21] Vulchanova M, Vulchanov V, Stankova M. Childhood Autism Spectrum Test (CAST), Bulgarian adaptation 2012. Available from: http://www.autismresearchcentre.com/arc_tests

[22] Constantino JN, Gruber CP, Davis S, Hayes S, Passanante $\mathrm{N}$, Przybeck T. The factor structure of autistic traits. Journal of Child Psychology and Psychiatry 2004; 45(4): 719-726. http://dx.doi.org/10.1111/j.1469-7610.2004.00266.x

[23] Kubinger K. On artificial results due to using factor analysis for dichotomous variables. Psychology Science 2003; 45(1): 106-110.

[24] Hurley AE, Scandura TA, Schriesheim CA, Brannick MT, Seers A, Vandenberg RJ. Exploratory and confirmatory factor analysis: Guidelines, issues, and alternatives. Journal of Organizational Behavior 1997; 18: 667-683.

http://dx.doi.org/10.1002/(SICI)10991379(199711)18:6<667::AID-JOB874>3.0.CO;2-T

[25] Stevens J. Applied multivariate statistics for the social sciences. Mahwah, NJ: Lawrence Erlbaum Associates, Inc. 2002.

[26] Torgerson WS. Theory and methods of scaling. New York: John Wiley and Sons, Inc. 1958.

[27] Kim JO, Mueller CW. Factor analysis: Statistical methods and practical issues. Newbury Park, Ca: Sage Publications, Inc.; 1978.

[28] Lord FM. Applications of Item response theory to practical testing problems. Hillsdale, NJ: Lawrence Erlbaum Associates, Inc.; 1980.

[29] O'Connor BP. SPSS and SAS programs for determining the number of components using parallel analysis and Velicer's MAP test. Behavior Research Methods, Instruments, and Computers 2000; 32: 396-402.

http://dx.doi.org/10.3758/BF03200807

[30] Revelle W. An overview of the psych package. Department of Psychology, Northwestern University; 2011; Available from: http://personality-project.org/r/overview.pdf

[31] Cattell RB. The scree test for the number of factors. Multivariate Behavioral Research 1966; 1: 245-276. http://dx.doi.org/10.1207/s15327906mbr0102 10

[32] Reynolds C, Kamphaus R, Eds. Handbook of psychological and Educational assessment of children. New York, NY: The Guilford Press 2003.

[33] Horn JL. A rationale and test for the number of factors in factor analysis. Psychometrika 1965; 30: 179-185. http://dx.doi.org/10.1007/BF02289447
[34] Buja A, Eyuboglu N. Remarks on parallel analysis. Multivariate Behavioral Research 1992; 27: 509-540. http://dx.doi.org/10.1207/s15327906mbr2704 2

[35] Costello AB, Osborne JW. Best practices in exploratory factor analysis: four recommendations for getting the most from your analysis. Practical Assessment Research and Evaluation 2005; 10(7): 1-9.

[36] Steiger $\mathrm{JH}$. Measures of fit in structural equation modeling: An introduction. 2009; Available from: http://www.statpower net/Content $/ 312 /$ Handout/Measures $\% 20$ of $\% 20$ Fit $\% 20$ in $\% 20$ Structural\%20Equation\%20Modeling.pdf

[37] Reckase MD. Unifactor latent trait models applied to multifactor tests: results and implications. Journal of Educational Statistics 1979; 4(3): 207-230. http://dx.doi.org/10.2307/1164671

[38] Cooke DJ, Michie C, Hart SD, Hare RD. Evaluating the screening version of the Hare Psychopathy Checklist Revised (PCL: V): An item response theory analysis. Psychological Assessment 1999; 11(1): 3-13. http://dx.doi.org/10.1037/1040-3590.11.1.3

[39] Pollard B, Dixon D, Dieppe P, Johnston M. Measuring the ICF components of impairment, activity limitation and participation restriction: an item analysis using classical test theory and item response theory. Health and Quality of Life Outcomes 2009; 7(41). http://dx.doi.org/10.1186/1477-7525-7-41

[40] Riekert KA, Eakin M. Factor analysis: A primer for asthma researchers. Journal of Allergy and Clinical Immunology 2008; 121: 1181-1183 http://dx.doi.org/10.1016/j.jaci.2008.03.023

[41] Mandy W, Charman T, Skuse D. Testing the Construct Validity of Proposed Criteria for DSM-5 Autism Spectrum Disorder. Journal of the American Academy of Child and Adolescent Psychiatry 2012; 51(1): 41-50. http://dx.doi.org/10.1016/j.jaac.2011.10.013

[42] Thurstone LL. The vectors of mind. Psychological Review 1934; 41: 1-32. http://dx.doi.org/10.1037/h0075959

[43] Thurstone LL. The vectors of mind: Multiple-factor analysis for the isolation of primary traits. Chicago: University of Chicago Press 1935 http://dx.doi.org/10.1037/10018-000

[44] Thurstone LL. The factorial isolation of primary abilities. Psychometrika 1936; 1: 175-182. http://dx.doi.org/10.1007/BF02288363

[45] Thurstone LL. Multiple-factor analysis. Chicago: University of Chicago Press 1947.

[46] Thorndike RM. Method of factor extraction and simple structure of data from diverse scientific areas. Paper presented at the annual meeting of the Western psychological association, San Francisco, CA. 1971

[47] Harman HH. Modern factor analysis. Chicago: The University of Chicago Press 1976.

[48] Guthrie W, Swineford L, Nottke C, Wetherby A. Early diagnosis of autism spectrum disorder: stability and change in clinical diagnosis. Journal of Child Psychology and Psychiatry 2013; 54(5): 582-590. http://dx.doi.org/10.1111/jcpp.12008

\section{DOI: http://dx.doi.org/10.6000/2292-2598.2016.04.02.5}

(C) 2016 Vulchanova et al.; Licensee Lifescience Global.

This is an open access article licensed under the terms of the Creative Commons Attribution Non-Commercial License (http://creativecommons.org/licenses/by-nc/3.0/) which permits unrestricted, non-commercial use, distribution and reproduction in any medium, provided the work is properly cited. 